\title{
Statistical Prediction of Ocean Circulation and Trajectories
}

\author{
Greg Holloway \\ Institute of Ocean Sciences, Sidney, BC V8L 4B2 Canada \\ phone: (250)363-6564 fax: (250)363-6746 email: hollowayg@ @ac.dfo-mpo.gc.ca \\ William Merryfield \\ Institute of Ocean Sciences, Sidney, BC V8L 4B2 Canada \\ phone: (250)363-6402 fax: (250)363-6746 email: merryfieldw@pac.dfo-mpo.gc.ca \\ Award \#: N000149910050 \\ http://www-sci.pac.dfo-mpo.gc.ca/osap/projects/jpod/jpod.htm
}

\section{LONG-TERM GOALS}

Our goals are to develop efficient means for statistically forecasting ocean currents and Lagrangian trajectories.

\section{OBJECTIVES}

Objectives are to formulate and test methods for predicting flows and trajectories that represent evolution of uncertainty and feedback of the uncertain component upon the mean. Such statistical predictions can be obtained via ensemble forecasts, but these are computationally expensive and suffer from statistical noise due to finite ensemble size. We seek statistical forecast methods that avoid these difficulties, guided by statistical mechanical theory and numerical investigation of quasi-geostrophic (qg) flow statistics. Anticipating application to more realistic flows, we have sought to extend statistical mechanics beyond qg to the case of finite-amplitude topography.

\section{APPROACH}

We approach the statistical forecast problem by considering the evolving probability density function (pdf). Direct computation of pdf evolution via the Liouville equation is intractable, so we consider equations for evolving moments of the pdf. The moment equations contain fluxes that generate uncertainty and drive the pdf toward higher-entropy configurations. These fluxes are akin to the irreversible (entropy-generating) fluxes encountered in microscopic systems.

Guided by statistical mechanics, we treat irreversible flux terms as generalized forces that drive the system toward higher entropy in accord with $H$-theorems for chaotic fluid systems (e.g. Carnevale, et al. 1981; Carnevale and Holloway 1982). The resulting equations predict evolution of the mean and of the spatially-dependent variance about the mean, which quantifies uncertainty. In the case of Lagrangian trajectories, we predict the evolving physical-space pdf, including effects of initial uncertainty in position and of the evolving uncertainty in the flow field.

As a testbed we consider a single-layer quasi-geostrophic flow over topography, whose equilibrium statistics are known (Salmon, et al. 1976). Moment equation forecast skill is evaluated by comparing 


\section{Report Documentation Page}

Form Approved

OMB No. 0704-0188

Public reporting burden for the collection of information is estimated to average 1 hour per response, including the time for reviewing instructions, searching existing data sources, gathering and maintaining the data needed, and completing and reviewing the collection of information Send comments regarding this burden estimate or any other aspect of this collection of information,

including suggestions for reducing this burden, to Washington Headquarters Services, Directorate for Information Operations and Reports, 1215 Jefferson Davis Highway, Suite 1204, Arlington

VA 22202-4302 Respondents should be aware that notwithstanding any other provision of law, no person shall be subject to a penalty for failing to comply with a collection of information if it

does not display a currently valid OMB control number

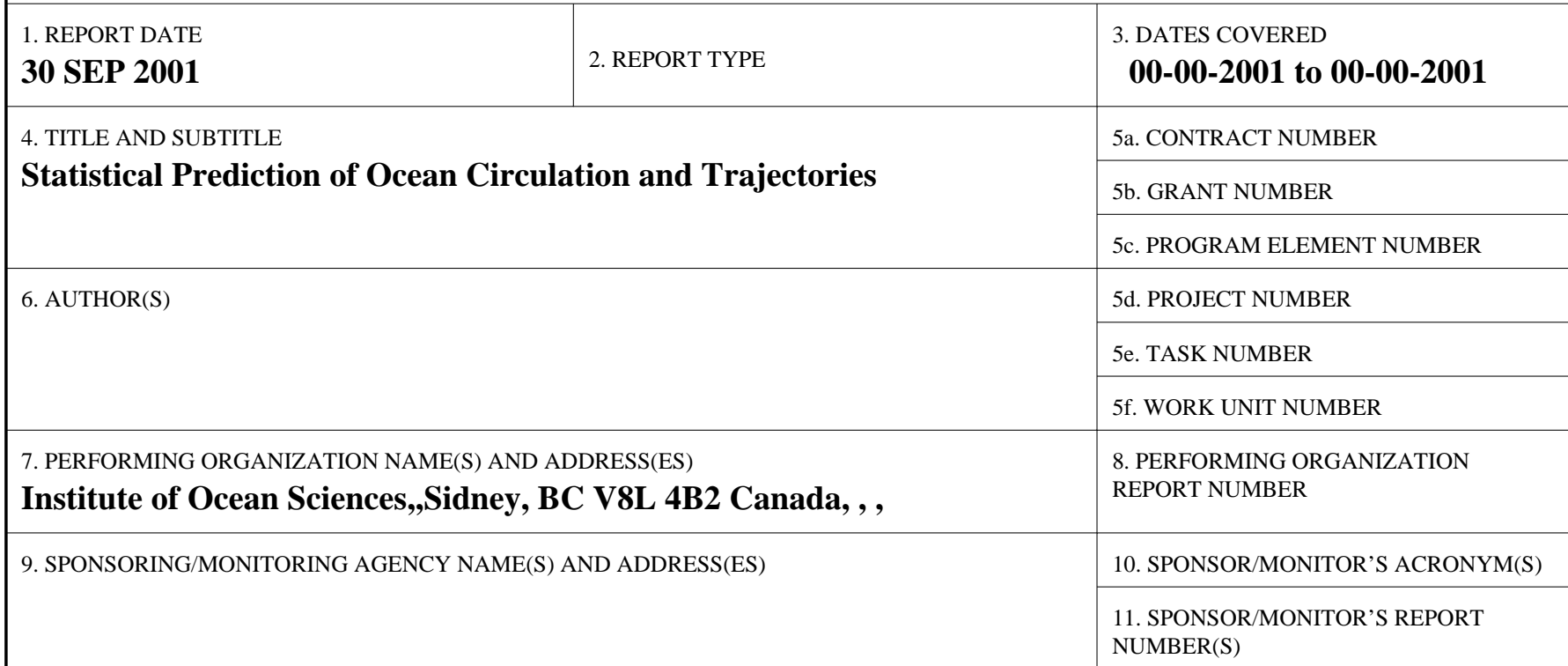

12. DISTRIBUTION/AVAILABILITY STATEMENT

Approved for public release; distribution unlimited

13. SUPPLEMENTARY NOTES

14. ABSTRACT

Objectives are to formulate and test methods for predicting flows and trajectories that represent evolution of uncertainty and feedback of the uncertain component upon the mean. Such statistical predictions can be obtained via ensemble forecasts, but these are computationally expensive and suffer from statistical noise due to finite ensemble size. We seek statistical forecast methods that avoid these difficulties, guided by statistical mechanical theory and numerical investigation of quasi-geostrophic (qg) flow statistics. Anticipating application to more realistic flows, we have sought to extend statistical mechanics beyond qg to the case of finite-amplitude topography.

\section{SUBJECT TERMS}

16. SECURITY CLASSIFICATION OF:

a REPORT unclassified b ABSTRACT unclassified c THIS PAGE unclassified
17. LIMITATION OF ABSTRACT

Same as Report (SAR)
18. NUMBER OF PAGES

6 19a. NAME OF RESPONSIBLE PERSON 

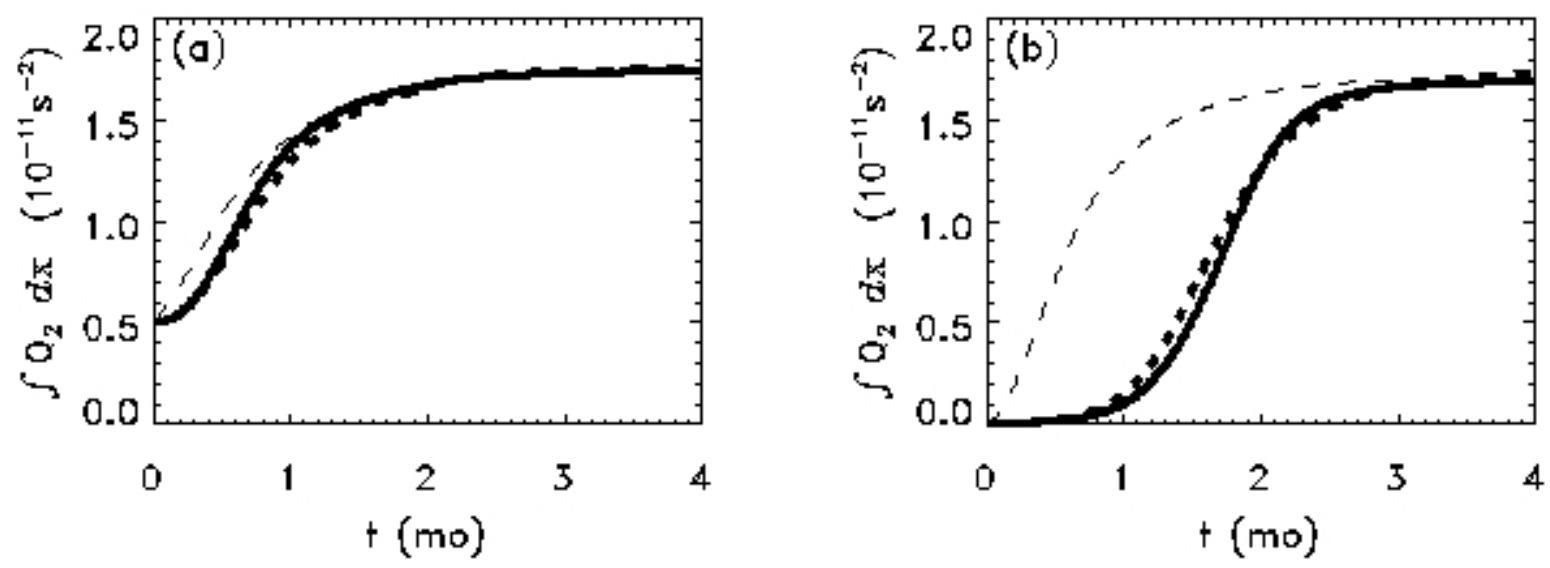

Figure 1. Total uncertainty vs. time for turbulent quasi-geostrophic flow in which initial uncertainty is relatively large (a) and very small (b). Solid curves: explicit $10^{4}$ member ensemble ("truth"); thick dashed curves: moment forecast accounting for initial development of irreversible fluxes; thin dashed curves: moment forecasts not accounting for initial development of irreversible fluxes. The more detailed treatment improves forecast performance, especially when initial uncertainty is small as in (b).

with various ensemble forecasts, with "truth" approximated by very large ensembles of up to $10^{4}$ realizations. We work in the spatial (rather than spectral) domain to facilitate application to ocean models

\section{WORK COMPLETED}

The method developed previously for predicting moments of qg flows was extended to represent an initial adjustment phase. It was shown that when initial errors are random, irreversible fluxes of mean potential vorticity and uncertainty initially vanish, and subsequently grow at a rate given approximately by the fluctuation energy density times gradients of the respective moments. Moment forecasts described in Merryfield and Holloway (2001a) were repeated using the modified scheme and reported in a revised version of that paper.

Coupled moment prediction equations for flow statistics and Lagrangian pdf were obtained, and a code was written to integrate these coupled equations numerically. The very accurate advection algorithm of Prather (1986) was adapted to represent positive-definite evolution of Lagrangian pdf with minimal numerical diffusion. Model integrations were performed and comparisons undertaken between moment equation predictions and explicit ensembles containing from 1 to $10^{3}$ realizations. Each set of experiments considered flow in a $1280 \mathrm{~km}$ square periodic domain, with initial rms velocity of $10 \mathrm{~cm} \mathrm{~s}^{-1}$ and eddy length scales of order $100 \mathrm{~km}$. Forecasts assuming uncertain initial flow and Lagrangian position were run for 4 months. A publication describing this work is in preparation.

As a contribution to a parallel Arctic modelling effort, the Prather (1986) advection algorithm employed in our Lagrangian prediction code was applied to sea-ice advection in an Arctic circulation model. A paper describing sensitivity to this scheme was submitted (Merryfield and Holloway 2001b). 

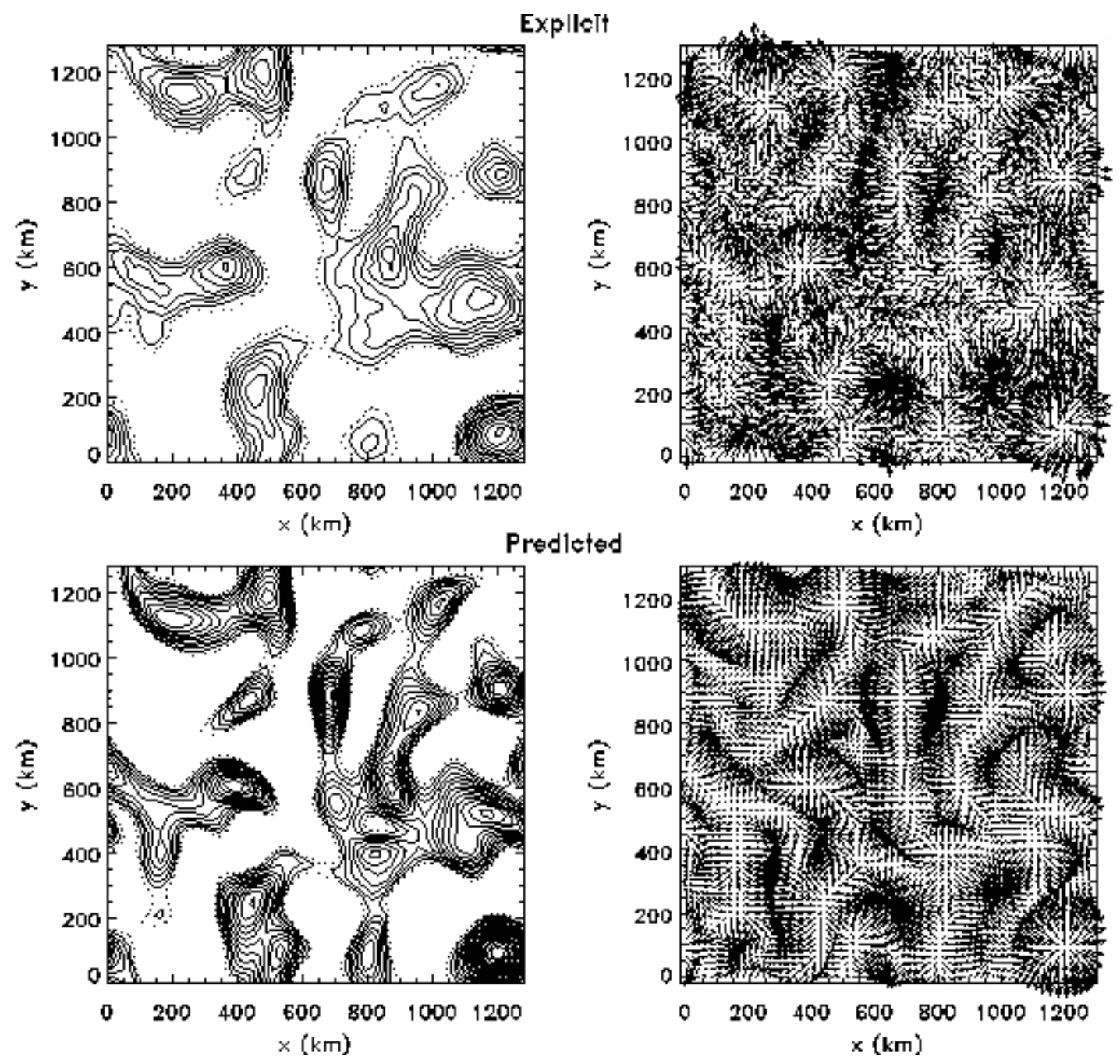

Figure 2. Left: Mean potential vorticity fields from explicit $10^{4}$ member ensemble (a) and moment forecast (c) at $t=2$ months. Contour interval is $0.83 \times 10^{-6} \mathrm{~s}^{-1}$; for clarity, only positive contours are shown. Right: Irreversible fluxes of mean potential vorticity, from explicit ensemble (b) and moment forecast $(d)$.

\section{RESULTS}

Forecast skill was significantly improved by the modification to the moment prediction equations to more accurately describe initial development of irreversible fluxes. Figure 1 shows evolution of spatially integrated uncertainty (pv variance $Q_{2}$ about the mean) versus time for cases in which initial uncertainty is relatively large (left) and small (right). True evolution (solid curves) is better predicted by the modified forecast procedure (thick dashed curves) than previously (thin dashed curves), especially when initial uncertainty is small.

The coupled moment prediction equations for flow statistics and Lagrangian pdf exhibited skill comparable to that of large ensembles. Figure 2 compares explicit $\left(10^{4}\right.$ realization ensemble $)$ and forecast mean pv (left) as well as the corresponding irreversible fluxes (right). Figure 3 compares 

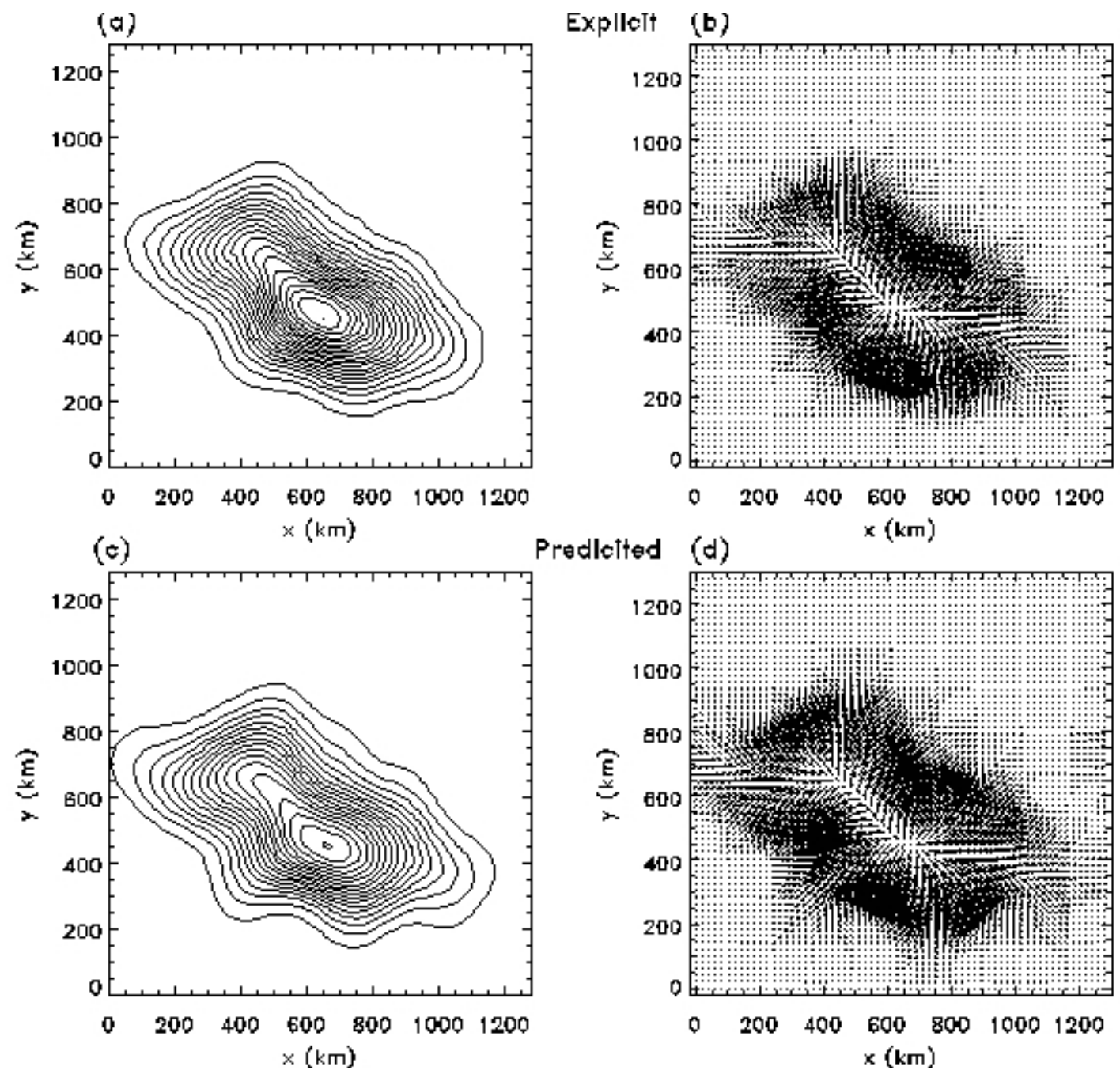

Figure 3. Left: Lagrangian pdf from explicit ensemble (a) and moment equation forecast (c), at $t=2$ months for case shown in Fig. 2. Initially the pdf was a Gaussian of half-width $40 \mathrm{~km}$, centered in the domain. Right: Irreversible fluxes of Lagrangian pdf, from explicit ensemble (b) and moment equation forecast $(d)$.

explicit and forecast Lagrangian pdf (left) and corresponding irreversible fluxes (left) for the flow field shown in Figure 2. In this case the Lagrangian pdf initially is a Gaussian spot concentrated in the center of the domain.

\section{IMPACT/APPLICATIONS}

Practical implementation of a moment equation scheme would enable statistical prediction without costly ensemble integrations. Application of this technique to Lagrangian prediction enables forecasts that account for uncertainty of the flow as well as initial uncertainty in position. 


\section{TRANSITIONS}

An updated version of the neptune eddy parameterization that incorporates effects of stratification and finite topography as proposed in Merryfield et al. (2001) is being implemented in an Arctic circulation model.

\section{RELATED PROJECTS}

Holloway leads an Arctic ice/ocean/atmosphere modeling effort as part of an Ocean Climate initiative by the Department of Fisheries and Oceans. Funding to Holloway is 60k/year (Canadian \$) for two years with notional commitment to a third year. This work reinforces our ONR-funded research by advancing the state of ocean models used to implement and test subgrid-scale parameterizations.

\section{REFERENCES}

Carnevale, G. F., U. Frisch, and R. Salmon, 1981: $H$ theorems in statistical fluid dynamics. J. Phys. A, 14, 1701-1718.

Carnevale, G. F., and G. Holloway, 1982: Information decay and the predictability of turbulent flows. J. Fluid Mech., 116, 115-121.

Salmon, R., G. Holloway, and M. C. Hendershott, 1976: The equilibrium statistical mechanics of simple quasi-geostrophic models. J. Fluid Mech., 75, 691-703.

\section{PUBLICATIONS}

Merryfield, W. J. and G. Holloway, 2001a: Predictability of quasi-geostrophic turbulence. J. Fluid Mech., submitted.

Merryfield, W. J., P. C. Cummins, and G. Holloway, 2001: Equilibrium statistical mechanics of barotropic flow over finite topography. J. Phys. Oceanogr., 31,1880-1890.

Merryfield, W. J., 2001: Intrusions in double-diffusively stable Arctic waters: Evidence for differential mixing? J. Phys. Oceanogr., in press.

Merryfield, W. J. and G. Holloway, 2001b: Application of a highly-accurate advection algorithm to sea-ice modelling. Ocean Modelling, submitted.

Holloway, G. and T. Sou, 2001: Has Arctic sea ice rapidly thinned? J. Climate, submitted.

Holloway, G., Gargett, A. E. and Merryfield, W. J., 2001: Differential diffusion in bistable conditions, in Proc. $12^{\text {th }}$ 'Aha Huliko'a Hawaiian Winter Workshop, From Stirring to Mixing in a Stratified Ocean, Honolulu, January 2000. 